\title{
Clinical Application of Liver Stiffness Measurement for Assessing the Risk of Hepatocellular Carcinoma
}

\author{
Jeong-Ju Yoo ${ }^{1 *}$, Eun-Ae Jung ${ }^{2^{*}}$, Sang Gyune Kim \\ ${ }^{1}$ Division of Gastroenterology and Hepatology, Department of Internal Medicine, Soonchunhyang University College of Medicine; ${ }^{2}$ Medi- \\ cal Library, Soonchunhyang University Bucheon Hospital, Bucheon, Korea
}

Received Fed. 8, 2019

Revised Feb. 25, 2019

Accepted Feb. 25, 2019
The most significant risk factor for hepatocellular carcinoma (HCC) is the presence of cirrhosis or advanced fibrosis of the liver. Liver biopsy was traditionally considered the gold standard for assessing the liver fibrosis burden. Recently, non-invasive methods, particularly transient elastography (TE), have proven effective at measuring fibrosis and determining cirrhosis. Clinical application of TE ranges from measuring fibrosis to predicting long-term prognosis and treatment response. Here, we focus on recent studies on the prognostic value of TE for predicting HCC. (J Liver Cancer 2019;19:12-18)

Keywords: Hepatocellular carcinoma; Transient elastography; Liver stiffness; Liver fibrosis; Liver cirrhosis

\section{INTRODUCTION}

The degree of fibrosis in patients with chronic liver disease (CLD) is a well-known prognostic factor. ${ }^{1}$ Historically, liver biopsy has been the standard method for evaluating liver fibrosis. However, this method is not widely used in clinical practice because of its invasiveness, diversity of sampling, and diagnostic variability. ${ }^{2}$ Several non-invasive methods have been studied to overcome these limitations. Serum fibrosis markers or prediction algorithms have shown relatively good predictive power; however, ultrasound elastography using shear wave elastography (SWE) or magnetic resonance

\section{Corresponding author : Sang Gyune Kim}

Department of Gastroenterology and Hepatology, Digestive Research Center and Liver Clinic, Soonchunhyang University Bucheon Hospital, 170 Jomaru-ro, Bucheon 14584, Korea

Tel. +82-32-621-5215, Fax. +82-32-621-6079

E-mail; mcnulty@schmc.ac.kr

https://orcid.org/0000-0001-8694-777X elastography (MRE) has almost replaced liver biopsy in the last decade. ${ }^{3}$ In particular, transient elastography (TE) (FibroScan ${ }^{\oplus}$; Echosens, Paris, France) is the most widely available liver fibrosis measurement method in clinical practice. Many studies have reported that liver stiffness measurements (LSMs) using TE can accurately predict liver fibrosis. ${ }^{4}$ In recent years, SWE has been used to measure liver stiffness (LS) in real time during ultrasonography. ${ }^{5} \mathrm{MRE}$, the most accurate method for evaluating liver fibrosis, has been widely used in various clinical trials as a replacement for liver biopsy. ${ }^{6}$

The recent use of these tools has expanded to predict longterm prognosis or the occurrence of hepatocellular carcinoma (HCC). Many studies have shown that these methods might be effective for predicting HCC and assessing fibrosis. Here we focused on recent studies of the prognostic value of non-invasive methods, mainly TE, for predicting HCC.

*Yoo JJ and Jung EA contributed equally as co-first authors. 


\section{TRANSIENT ELASTOGRAPHY}

TE is the most widely used non-invasive method, and many studies have examined the association between LSM and HCC. Although most studies have focused on patients with viral hepatitis, LSM is also useful for predicting HCC recurrence after resection or radiofrequency ablation (RFA).

\section{Hepatitis C virus}

Table 1 summarizes studies on the prediction of HCC incidence using TE in patients with chronic hepatitis $\mathrm{C}(\mathrm{CHC})$. In 2009, a study by Masuzaki et al. ${ }^{7}$ was the first to report that TE may aid in predicting the occurrence of HCC in $\mathrm{CHC}$ patients. This study showed that a higher LS value was a significant risk factor for HCC development, especially measurements of LS value $>10$ kilopascal $(\mathrm{kPa})$ (hazard ratio $[\mathrm{HR}], 16.7$; 95\% confidence interval [CI], 3.71-75.2; $P<0.001)$. Moreover, among those with a higher LS value, the incidence of HCC increased in proportion to the LS value value. Therefore, they suggested that the usefulness of LS value was not limited to a surrogate for liver biopsy but can be applied as an indicator of HCC development.

Thereafter, studies focused on predicting the incidence of HCC after interferon (IFN) therapy or administration of direct acting antivirals (DAAs). In 2014, Narita et al. ${ }^{8}$ found that a high LS value ( $>14.0 \mathrm{kPa}$ ) was an independent risk factor for HCC development (HR, 5.58; $P=0.020)$. Furthermore, the combination of three risk factors-LS value, platelet count, and non-sustained virologic response (SVR)-were effectively stratified to predict the risk of HCC in patients receiving IFN therapy. Since DAAs have replaced IFN therapy, two studies have described patients taking DAAs. A large prospective cohort study conducted in Japan recruited 263 patients treated with DAAs in whom acoustic radiation force impulse was used to measure LS. ${ }^{9}$ The study reported that the incidence of HCC was relatively high in patients with a high baseline LS value, even if SVR was achieved after administration of DAAs. Therefore, a higher LS value $(>1.73$ $\mathrm{m} / \mathrm{s}$ ) at baseline was an independent predictor of HCC occurrence (HR, 8.35; 95\% CI, 1.62-43.09; $P=0.011$ ). Thus, careful surveillance after DAA treatment is still required in patients with a higher baseline LS value. In 2018, Ravaioli et al. ${ }^{10}$ suggested that baseline LS value and decrease in LS value after DAA treatment are equally important. An LS value reduction (delta LS) of lower than -30\% was independently associated with HCC development (HR, 5.36; 95\% CI, 1.56$18.40 ; P=0.008)$.

\section{Hepatitis $B$ virus}

Predicting the occurrence of HCC using TE in patients with chronic hepatitis $\mathrm{B}(\mathrm{CHB})$ has been the most highly studied field, mainly in Korea. This is thought to be due to the high incidence of hepatitis B virus (HBV) in Northeast Asia, unlike hepatitis $\mathrm{C}$ virus (HCV), which has a high incidence in Europe and Japan. Table 2 summarizes studies of the prediction of HCC incidence using TE in CHB patients. Most of the studies recruited large-scale patients.

In 2011, Jung et al. ${ }^{11}$ published a prospective cohort study of 1,130 non-biopsy-proven CHB patients. In a multivariate analysis, patients with a higher LSM $(>8 \mathrm{kPa})$ were at significantly greater risk of HCC development. Furthermore, the incidence of HCC increased as LS values increased (LS value,

Table 1. Prediction of hepatocellular carcinoma incidence in patients with chronic hepatitis $C$

\begin{tabular}{|c|c|c|c|c|c|c|c|c|}
\hline Year & Author & Tool & Patients & Study type & Design & Number & $\begin{array}{l}\text { Duration } \\
\text { (months) }\end{array}$ & $\begin{array}{l}\text { Incidence } \\
\text { of HCC }\end{array}$ \\
\hline 2018 & Ravaioli et al ${ }^{10}$ & $\mathrm{TE}$ & $\begin{array}{l}\text { Liver cirrhosis treated with direct } \\
\text { acting antiviral }\end{array}$ & Retrospective & Cohort study & 139 & 15 & $14.4 \%$ \\
\hline 2017 & Tachi et al $^{9}$ & ARFI & Treated with direct-acting antiviral & Prospective & Cohort study & 263 & 18.1 & $7.2 \%$ \\
\hline 2014 & Narita et al ${ }^{8}$ & $\mathrm{TE}$ & Treated with interferon & Retrospective & Cohort study & 151 & 24 & $6 \%$ \\
\hline 2009 & Masuzaki et al ${ }^{7}$ & $\mathrm{TE}$ & HCV RNA positive & Prospective & Cohort study & 866 & 36 & $8.9 \%$ \\
\hline
\end{tabular}

HCV, hepatitis C virus; HCC, hepatocellular carcinoma; TE, transient elastography; ARFI, acoustic radiation force impulse; RNA, ribonucleic acid. 
8.1-13 kPa, HR, 3.07; LS value, 13.1-18 kPa, HR, 4.68; LS value, 18.1-23 kPa, HR, 5.55; LS value >23 kPa, HR, 6.60). Therefore, this is the first report in $\mathrm{CHB}$ patients to suggest that LSM could be a useful predictor of HCC development.

In 2014, Wong et al. ${ }^{12}$ developed a model for predicting the incidence of HCC in 1,555 CHB patients; LSM was one of the most important risk factors. In the training cohort, the occurrence of HCC was increased about 3.9 times when LS value was higher than $8 \mathrm{kPa}(\mathrm{HR}, 3.9$; 95\% CI, 1.5-9.7; $P=0.004)$. When LS value was higher than $12 \mathrm{kPa}$, the risk of HCC increased by about 6 times (HR, 6.0; 95\% CI, 2.5-14.6; $P<0.001)$.

In 2015, a study on patients with TE-defined subclinical cirrhosis and an LS value more than $13 \mathrm{kPa}$ was published. ${ }^{13}$ The incidence of HCC was significantly higher in those with subclinical cirrhosis than in those without cirrhosis (13.3 per 1,000 person-years vs. 3.4 per 1,000 person-years). In addition, subclinical cirrhosis was independently associated with a risk of HCC incidence regardless of antiviral therapy (without antiviral therapy: HR, 4.68; with antiviral therapy: HR, 3.34). Therefore, TE was an effective tool for distinguishing between cases of subclinical cirrhosis that were not clinically cirrhosis but had a high risk of HCC development. A study of an LS group in the subcirrhotic range, which was defined as LS values less than $13 \mathrm{kPa}$, was reported in the same group in 2017. ${ }^{14}$ The comparison of 214 patients with subcirrhotic range LS value with 540 patients with definitive clinical cirrhosis revealed that the cumulative HCC incidence was significantly lower in patients with subcirrhotic LS value (HR, $0.462 ; P<0.05)$. Therefore, the authors concluded that different TE-based HCC surveillance strategies may be required.

In 2015, Shin et al. ${ }^{15}$ predicted HCC risk using an LSMbased prediction model, LSPS (LS value $\times$ spleen diameter/ platelet count), in 227 patients with $\mathrm{CHB}$ who underwent liver biopsy. LSPS was originally used to predict portal hypertension-related complications, but it was also effective at predicting $\mathrm{HCC}$ in $\mathrm{CHB}$ patients.

In 2015, Kim et al. ${ }^{13}$ compared the predictive power of TE and fibrosis-4 (FIB-4) score in 1,308 CHB patients. LSM showed significantly better performance than FIB-4 at assessing the risk of HCC development, but the combined use of LSM and FIB-4 did not provide an additional benefit compared to LS alone. Thus, the authors concluded that LSM alone might be helpful for optimizing HCC surveillance strategies.

In 2016, Seo et al. ${ }^{16}$ performed TE using liver biopsy sam-

Table 2. Prediction of hepatocellular carcinoma incidence in patients with hepatitis B virus

\begin{tabular}{|c|c|c|c|c|c|c|c|c|}
\hline Year & Author & Tool & Patients & Study type & Design & Number & $\begin{array}{l}\text { Duration } \\
\text { (months) }\end{array}$ & $\begin{array}{l}\text { Incidence } \\
\text { of HCC }\end{array}$ \\
\hline 2018 & Song et $\left.a\right|^{18}$ & TE & Antiviral therapy more than 1 year & Retrospective & Cohort study & 1,014 & 44.4 & $3.6 \%$ \\
\hline 2018 & Kim et $\mathrm{al}^{17}$ & $\mathrm{TE}$ & $\begin{array}{l}\text { Advanced fibrosis or cirrhosis taking } \\
\text { antiviral therapy }\end{array}$ & Retrospective & Cohort study & 209 & 62.2 & $13.4 \%$ \\
\hline 2017 & Jeon et al ${ }^{14}$ & TE & $\begin{array}{l}\text { Chronic hepatitis B-related cirrhosis or } \\
\text { subclinical cirrhosis }\end{array}$ & Retrospective & Cohort study & 540 & 54.1 & $15.0 \%$ \\
\hline 2016 & Li et $\left.a\right|^{28}$ & TE & Cirrhotic nodule and small HCC & Retrospective & Cross-sectional & 95 & $(-)$ & $(-)$ \\
\hline 2016 & Seo et $\mathrm{al}^{16}$ & TE & Initiating antiviral therapy & Retrospective & Cohort study & 381 & 48.1 & $8.9 \%$ \\
\hline 2016 & Liu et $\mathrm{al}^{29}$ & TE & $\begin{array}{l}\text { Previously untreated HBV related liver } \\
\text { cirrhosis }\end{array}$ & Retrospective & Cross-sectional & 156 & $(-)$ & $(-)$ \\
\hline 2016 & Kim et $a 1^{30}$ & TE & Chronic hepatitis B & Retrospective & Cohort study & 1,308 & 73.2 & $9.1 \%$ \\
\hline 2015 & Shin et $a 1^{15}$ & TE & Chronic hepatitis B with liver biopsy & Retrospective & Cohort study & 227 & 61.7 & $7.9 \%$ \\
\hline 2015 & Kim et $\mathrm{al}^{13}$ & TE & $\begin{array}{l}\text { Chronic hepatitis B without clinically } \\
\text { liver cirrhosis }\end{array}$ & Prospective & Cohort study & 2,876 & 48.9 & $1.81 \%$ \\
\hline 2014 & Wong et $a l^{12}$ & $\mathrm{TE}$ & Chronic hepatitis B & Prospective & Cohort study & 1,555 & 69 & $3.5 \%$ \\
\hline 2011 & Jung et al ${ }^{11}$ & $\mathrm{TE}$ & Non-biopsy proven chronic hepatitis B & Prospective & Cohort study & 1,130 & 30.7 & $5.0 \%$ \\
\hline
\end{tabular}

HBV, hepatitis B virus; HCC, hepatocellular carcinoma; TE, transient elastography. 
ples in 381 patients who took antiviral drugs. In this study, LSM was reportedly more effective at predicting HCC than the histological staging of the liver biopsy sample itself. LS value was an independent predictor of HCC development (HR, 1.04; $P<0.001$ ), whereas histological staging was not $(P>0.05)$. This is probably because the TE value reflects both fibrosis and inflammation, which is closely associated with viral activity.

In 2018, Kim et al. ${ }^{17}$ published a study of 209 patients with CHB-related advanced fibrosis or cirrhosis who received paired TE examinations during antiviral therapy. They found that the risk of HCC was reduced when the LS value was lowered to the subcirrhotic range after antiviral agent use (HR, 0.485; $P=0.047$ ). This phenomenon was also observed in patients diagnosed with cirrhosis in a baseline biopsy. When LS value was maintained at the cirrhosis range after 2 years of antiviral use, the incidence of HCC was significantly higher than that of the decreased LS value group.

In 2018, Song et al. ${ }^{18}$ compared the predictive ability of TE, aspartate aminotransferase-to-platelet ratio (APRI), and FIB4 score to predict HCC incidence in 1,014 patients who took antiviral drugs for more than 1 year. Patients with low APRI or FIB-4 scores but high LS values ( $>6 \mathrm{kPa}$ ) were sub-analyzed in this study. In this group, the HCC risk was significantly different according to the LS value. Thus, LSM could play an additional role compared to serum biomarkers for stratifying the risk of HCC.

\section{Mixed etiology}

For studies that do not distinguish between etiologies, cross-sectional studies are most common. Table 3 summa- rizes studies to date of the prediction of HCC incidence using TE in mixed etiology.

In 2015, Tatsumi et al. ${ }^{19}$ compared 756 patients with CLD and 246 patients with HCC to determine the LSM cut-off that predicts the occurrence of HCC. For the HCV group, the cut-off was $12.0 \mathrm{kPa}$, while the cut-off for the HBV group was $8.5 \mathrm{kPa}$ and that for the non-B/non-C group was $12.0 \mathrm{kPa}$. In 2016, Adler et al. ${ }^{20}$ conducted a cohort study of $432 \mathrm{pa}-$ tients with cirrhosis with an LS value $>20 \mathrm{kPa}$. When LS values were classified into four groups, the risk of HCC increased in the group with a high LS value (20-25 kPa, HR, 1.0; 25-30 kPa, HR, 1.55; 30-40 kPa, HR, 3.21; >40 kPa, HR, 4.08). This study showed that TE is useful not only as a measure of fibrosis, but also for evaluating the risk of HCC, even in patients who already have cirrhosis.

In 2017, Sugihara et al. ${ }^{21}$ published a study in which TE was performed in the Japanese general population aged 40 years and older. In this cross-sectional study of 181 patients, only three patients had HCC. For detecting HCC, the cut-off value of LS value was $5.3 \mathrm{kPa}$, with a sensitivity of $100 \%$, specificity of $75 \%$, and area under the receiver operating characteristic curve of 0.88 ; these predictive powers were further increased when LSM and a controlled attenuation parameter (CAP) were combined. Although the number of HCC patients in this study was small, it is meaningful that the research was conducted of the general population.

\section{HCC recurrence after resection or RFA}

Previous studies have shown that LSM can predict postoperative failure. Recent studies have examined the relationship between TE and recurrence of HCC after RFA or resec-

Table 3. Prediction of hepatocellular carcinoma incidence in cases of mixed etiology

\begin{tabular}{|c|c|c|c|c|c|c|c|c|}
\hline Year & Author & Tool & Patients & Study type & Design & Number & $\begin{array}{l}\text { Duration } \\
\text { (months) }\end{array}$ & $\begin{array}{l}\text { Incidence } \\
\text { of HCC }\end{array}$ \\
\hline 2017 & Sugihara et al ${ }^{21}$ & $\mathrm{TE}$ & $\begin{array}{l}\text { General population more than } \\
40 \text { years old }\end{array}$ & Retrospective & Cross-sectional & 181 & $(-)$ & $(-)$ \\
\hline 2016 & Adler et $\mathrm{a}^{20}$ & $\mathrm{TE}$ & Patients with $L S M \geq 20 \mathrm{kPa}$ & Retrospective & Cohort study & 432 & 36 & 14.7 \\
\hline 2015 & Tatsumi et al ${ }^{19}$ & $\mathrm{TE}$ & Chronic liver disease & Retrospective & Cross-sectional & 1,002 & $(-)$ & $(-)$ \\
\hline
\end{tabular}

HCC, hepatocellular carcinoma; TE, transient elastography. 
tion. Table 4 summarizes studies to date of the prediction of HCC incidence using TE after resection or RFA.

In 2014, Jung et al. ${ }^{22}$ reported a prospective study of 139 patients who underwent curative resection. LS value was an independent predictor of late HCC recurrence (odds ratio, $1.063 ; 95 \% \mathrm{CI}, 1.011-1.119 ; P=0.017)$. In addition to TE, a predictive model using necroinflammatory activity grade IIIII, tumor multiplicity, and indocyanine green retention rate at 15 minutes (ICG R15) was developed. This TE-based predictive model effectively predicted HCC recurrence.

A retrospective study of 111 patients who underwent RFA was reported in $2015 .{ }^{23}$ When the LS value was higher than $13.0 \mathrm{kPa}$, the HCC recurrence rate was significantly increased (HR, 3.115, 95\% CI, 1.238-7.842; $P<0.05$ ) and mortality was also elevated (HR, 9.834, 95\% CI, 1.148-84.211; $P<0.05)$. In 2017, in addition to the previous study, 228 RFA patients were newly included. ${ }^{24}$ Similar to the previous study, late recurrence was significantly elevated when the LS value was higher than $13.0 \mathrm{kPa}$ (HR, 4.507; 95\% CI, 2.131-7.724; $P<0.001)$.

\section{LIMITATION OF TE FOR PREDICTING HCC AND TE-BASED PREDICTION MODEL}

As described above, TE can be an effective tool for predicting HCC. However, debate persists about whether TE alone is sufficient to determine the risk of $\mathrm{HCC} .{ }^{25}$ Although TE has shown excellent efficacy at evaluating fibrosis, there are other risk factors for HCC development (e.g., sex, family history, inflammation) ${ }^{26}$ Furthermore, TE has variation even in the same patient by as much as $1.5 \mathrm{kPa} .^{27}$ Thus, TE results should be interpreted in context with other clinical findings. To overcome the limitation of TE alone, studies of the combina- tion of TE and other tests or biomarkers have been performed. However, few had a prospective longitudinal design.

In a study of 1,555 CHB patients, age, albumin, and HBV DNA were significant predictors of HCC development in addition to LSM. ${ }^{12}$ The LSM-HCC score combining these four factors effectively predicted the occurrence of HCC. By applying the cut-off value of 11, almost all patients who are at risk for developing HCC in the future can be excluded. In a study of 227 CHB patients who underwent liver biopsy, spleen diameter and platelet count (in addition to LSM) were associated with the risk of HCC. ${ }^{15}$

In patients with $\mathrm{CHC}$, platelet count and the presence of SVR were important predictors of HCC development in addition to LSM. ${ }^{8}$ When all three risk factors were present, the incidence of HCC was $59.6 \%$, whereas the risk decreased to $8.2 \%$ in the absence of all risk factors. In patients who underwent curative resection, necroinflammatory activity grade IIIII, tumor multiplicity, and ICG R15, as noted above, affected late HCC recurrence after curative resection in addition to LSM. $^{22}$

\section{CONCLUSIONS}

Recent advances in non-invasive methods allow us to predict the occurrence of HCC more easily and accurately. Of the methods now available, TE is an excellent tool for assessing fibrosis degree, and its application has been extended to the prediction of HCC incidence. This usefulness of TE in predicting future HCC was also confirmed by many longitudinal and prospective studies. However, since TE alone currently has limited ability to predict HCC, careful interpretation and other integrating test results should be considered. More research is needed to determine whether tools other

Table 4. Prediction of hepatocellular carcinoma recurrence after resection or radiofrequency ablation

\begin{tabular}{|c|c|c|c|c|c|c|c|c|}
\hline Year & Author & Tool & Patients & Study type & Design & Number & $\begin{array}{l}\text { Duration } \\
\text { (months) }\end{array}$ & $\begin{array}{c}\text { Recurrence of } \\
\text { HCC }\end{array}$ \\
\hline 2017 & Lee et $\mathrm{al}^{24}$ & $\mathrm{TE}$ & Post-RFA & Retrospective & Cohort study & 228 & 24 & 54.8 \\
\hline 2015 & Lee et $\mathrm{al}^{23}$ & TE & Post-RFA & Retrospective & Cohort study & 111 & 22.4 & 42.3 \\
\hline 2014 & Jung et $\mathrm{al}^{22}$ & TE & Post-curative resection & Prospective & Cohort study & 139 & 24.5 & 31.7 \\
\hline
\end{tabular}

HCC, hepatocellular carcinoma; TE, transient elastography; RFA, radiofrequency ablation. 
than TE, e.g., SWE or MRE, can effectively predict HCC. Furthermore, it will be interesting to see whether elastography can be used to distinguish benign from malignant liver lesions. ${ }^{25}$ Finally, further research is needed to determine whether the surveillance strategy for HCC in patients with CLD should be modified according to LS value.

\section{Conflicts of Interest}

The authors have no conflicts to disclose.

\section{REFERENCES}

1. Bolondi L, Sofia S, Siringo S, Gaiani S, Casali A, Zironi G, et al. Surveillance programme of cirrhotic patients for early diagnosis and treatment of hepatocellular carcinoma: a cost effectiveness analysis. Gut 2001;48:251-259.

2. Lo RC, Kim H. Histopathological evaluation of liver fibrosis and cirrhosis regression. Clin Mol Hepatol 2017;23:302-307.

3. Wong GL. Prediction of fibrosis progression in chronic viral hepatitis. Clin Mol Hepatol 2014;20:228-236.

4. Singh S, Fujii LL, Murad MH, Wang Z, Asrani SK, Ehman RL, et al. Liver stiffness is associated with risk of decompensation, liver cancer, and death in patients with chronic liver diseases: a systematic review and meta-analysis. Clin Gastroenterol Hepatol 2013;11:1573-1584.e1-2;quiz e88-e89.

5. Kudo M. Prediction of hepatocellular carcinoma incidence risk by ultrasound elastography. Liver Cancer 2014;3:1-5.

6. Venkatesh SK, Yin M, Ehman RL. Magnetic resonance elastography of liver: technique, analysis, and clinical applications. J Magn Reson Imaging 2013:37:544-555.

7. Masuzaki R, Tateishi R, Yoshida H, Goto E, Sato T, Ohki T, et al. Prospective risk assessment for hepatocellular carcinoma development in patients with chronic hepatitis $C$ by transient elastography. Hepatology 2009;49:1954-1961.

8. Narita Y, Genda T, Tsuzura H, Sato S, Kanemitsu Y, Ishikawa S, et al. Prediction of liver stiffness hepatocellular carcinoma in chronic hepatitis C patients on interferon- based anti-viral therapy. J Gastroenterol Hepatol 2014;29:137-143.

9. Tachi $Y$, Hirai T, Kojima Y, Ishizu Y, Honda T, Kuzuya $T$, et al. Liver stiffness measurement predicts hepatocellular carcinoma development in patients treated with direct-acting antivirals. JGH Open 2017;1:44-49.

10. Ravaioli F, Conti F, Brillanti S, Andreone P, Mazzella G, Buonfiglioli $F$, et al. Hepatocellular carcinoma risk assessment by the measurement of liver stiffness variations in HCV cirrhotics treated with direct acting antivirals. Dig Liver Dis 2018;50:573-579.
11. Jung KS, Kim SU, Ahn SH, Park YN, Kim DY, Park JY, et al. Risk assessment of hepatitis B virus-related hepatocellular carcinoma development using liver stiffness measurement (FibroScan). Hepatology 2011;53:885-894.

12. Wong GL, Chan HL, Wong CK, Leung C, Chan CY, Ho PP, et al. Liver stiffness- based optimization of hepatocellular carcinoma risk score in patients with chronic hepatitis B. J Hepatol 2014;60:339345.

13. Kim MN, Kim SU, Kim BK, Park JY, Kim DY, Ahn SH, et al. Increased risk of hepatocellular carcinoma in chronic hepatitis $B$ patients with transient elastography- defined subclinical cirrhosis. Hepatology 2015;61:1851-1859.

14. Jeon MY, Lee HW, Kim SU, Heo JY, Han S, Kim BK, et al. Subcirrhotic liver stiffness by FibroScan correlates with lower risk of hepatocellular carcinoma in patients with HBV-related cirrhosis. Hepatol Int 2017;11:268-276.

15. Shin SH, Kim SU, Park JY, Kim DY, Ahn SH, Han KH, et al. Liver stiffness-based model for prediction of hepatocellular carcinoma in chronic hepatitis B virus infection: comparison with histological fibrosis. Liver Int 2015;35:1054-1062.

16. Seo YS, Kim MN, Kim SU, Kim SG, Um SH, Han KH, et al. Risk assessment of hepatocellular carcinoma using transient elastography vs. liver biopsy in chronic hepatitis B patients receiving antiviral therapy. Medicine (Baltimore) 2016;95:e2985.

17. Kim BS, Seo YS, Kim YS, Lee CH, Lee HA, Um SH, et al. Reduced risk of hepatocellular carcinoma by achieving a subcirrhotic liver stiffness through antiviral agents in hepatitis B virus-related advanced fibrosis or cirrhosis. J Gastroenterol Hepatol 2018;33:503510.

18. Song BG, Sinn DH, Chi S, Kim K, Kang W, Gwak GY, et al. Additional role of liver stiffness measurement in stratifying residual hepatocellular carcinoma risk predicted by serum biomarkers in chronic hepatitis B patients under antiviral therapy. Eur J Gastroenterol Hepatol 2018;30:1447-1452.

19. Tatsumi A, Maekawa S, Sato M, Komatsu N, Miura M, Amemiya F, et al. Liver stiffness measurement for risk assessment of hepatocellular carcinoma. Hepatol Res 2015;45:523-532.

20. Adler M, Larocca L, Trovato FM, Marcinkowski H, Pasha Y, TaylorRobinson SD. Evaluating the risk of hepatocellular carcinoma in patients with prominently elevated liver stiffness measurements by FibroScan: a multicentre study. HPB (Oxford) 2016;18:678-683.

21. Sugihara T, Koda M, Matono T, Okamoto K, Murawaki Y, Isomoto $\mathrm{H}$, et al. Risk assessment of hepatocellular carcinoma in general population by liver stiffness in combination with controlled attenuation parameter using transient elastography: a cross sectional study. Yonago Acta Med 2017;60:106-112.

22. Jung KS, Kim JH, Kim SU, Song K, Kim BK, Park JY, et al. Liver stiffness value- based risk estimation of late recurrence after curative 
resection of hepatocellular carcinoma: development and validation of a predictive model. PLoS One 2014;9:e99167.

23. Lee SH, Kim SU, Jang JW, Bae SH, Lee S, Kim BK, et al. Use of transient elastography to predict de novo recurrence after radiofrequency ablation for hepatocellular carcinoma. Onco Targets Ther 2015;8:347-356.

24. Lee YR, Park SY, Kim SU, Jang SY, Tak WY, Kweon YO, et al. Using transient elastography to predict hepatocellular carcinoma recurrence after radiofrequency ablation. J Gastroenterol Hepatol 2017;32:1079-1086.

25. Trotter JF. Elastography and the risk of hepatocellular carcinoma. Gastroenterol Hepatol (NY) 2018;14:108-110.

26. Gomaa Al, Khan SA, Toledano MB, Waked I, Taylor-Robinson SD. Hepatocellular carcinoma: epidemiology, risk factors and patho- genesis. World J Gastroenterol 2008;14:4300-4308.

27. Yoshioka K, Kawabe N, Hashimoto S. Transient elastography: applications and limitations. Hepatol Res 2008;38:1063-1068.

28. Li JW, Ling WW, Lu Q, Lu CL, He D, Luo Y. Liver stiffness and serum alpha- fetoprotein in discriminating small hepatocellular carcinoma from cirrhotic nodule. Ultrasound Q 2016;32:319-326.

29. Liu XY, Ma LN, Yan TT, Lu ZH, Tang YY, Luo X, et al. Combined detection of liver stiffness and C-reactive protein in patients with hepatitis B virus-related liver cirrhosis, with and without hepatocellular carcinoma. Mol Clin Oncol 2016;4:587-590.

30. Kim SU, Kim BK, Park JY, Kim do Y, Ahn SH, Song K, et al. Transient elastography is superior to FIB-4 in assessing the risk of hepatocellular carcinoma in patients with chronic hepatitis $B$. Medicine (Baltimore) 2016;95:e3434. 